\title{
La participación femenina en el tenis: She rallies y Girl Power Camps
}

\author{
Julie Gordon, Judy Murray y Emma Doyle
}

\section{RESUMEN}

Está ampliamente aceptado que en cuanto las niñas llegan a la pubertad, su participación deportiva declina rápidamente. Este artículo intenta resumir la literatura que explora los factores psicológicos relacionados con el éxito, y destacar dos iniciativas que se están llevando a cabo para atraer, comprometer y mantener a más mujeres en el tenis. Concluye con la evaluación de estas iniciativas dentro del contexto de la teoría del liderazgo transformacional, que puede ofrecer valiosa información, no solamente para el tenis, sino para el deporte en general.
Palabras clave: Niñas, participación, teoría del liderazgo transformacional, tenis

Recibido: 15 Feb 2017

Aceptado: 24 May 2017

Autor correspondiente: Julie Gordon

Correo electrónico:

juliegtennis@me.com

\section{INTRODUCCIÓN}

\section{Parte Uno - Antecedentes}

Gran cantidad de datos demuestran que a medida que las niñas se acercan a la adolescencia, se reduce su participación deportiva (Nader, Bradley, Houts, McRitchie, y O'Brien, 2008). Dado que la actividad física y la participación deportiva son esenciales para la salud y el bienestar, aumentar la participación femenina en la actividad física se considera una prioridad nacional e internacional (Davies, Burns, Jewell, y McBride, 2011; Organización Mundial de la Salud, 2006).

La campaña "Esta niña puede", desarrollada por Sport England, y diseñada para aumentar la participación femenina y la actividad física en el deporte, ha sido evaluada en un informe Ilamado "Ir a donde están las mujeres" (Sport England (2015). Este documento proporciona puntos de vista específicos sobre la efectividad de varias iniciativas para comprometer a niñas y mujeres en el deporte y la actividad física. Recomienda que las intervenciones consideren la necesidad de inspirar confianza, el uso de modelos de rol adecuados y el apoyo social, celebrando los logros regularmente y con sinceridad (Reading y cols., 2014).

En general, existe poca evidencia sobre las intervenciones efectivas para aumentar la participación femenina en el deporte per se (Priest, Armstrong, Doyle, y Waters, 2008). Sin embargo, hay evidencia sobre la efectividad de las intervenciones a la hora de aumentar la actividad física de las niñas, destacando los factores psicológicos clave que deben sustentar una intervención orientada a la mayor participación de las niñas en el deporte. Un meta-análisis reciente de la efectividad de las acciones para aumentar la actividad física entre niñas adolescentes (Biddle, Braithwaite, y Pearson, 2014) encontró que las intervenciones teóricas, en los colegios, solamente de niñas, utilizaban estrategias multi-componente, y eran más efectivas.

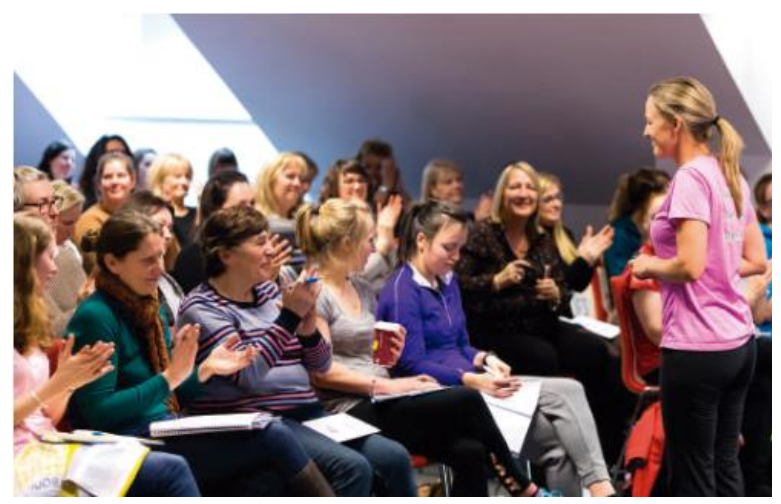

Las estrategias "multi-componente" son aquellas que incorporan, por ejemplo, a los colegios más la comunidad, más la familia, y añaden un elemento de apoyo social (Biddle y cols., 2014; Inchley, Mitchell, y Currie, 2012).

Resumiendo, a partir de la literatura sobre las niñas y la actividad física, surgen varios factores clave. Parecen sugerir que la intervención efectiva debe:

- inspirar confianza 
- utilizar una estrategia multi-componente

- proporcionar un entorno de apoyo

- proporcionar oportunidades fundamentalmente locales

- estar sustentada por la teoría

- ser de sexo único

- incorporar el uso de modelos de rol adecuados

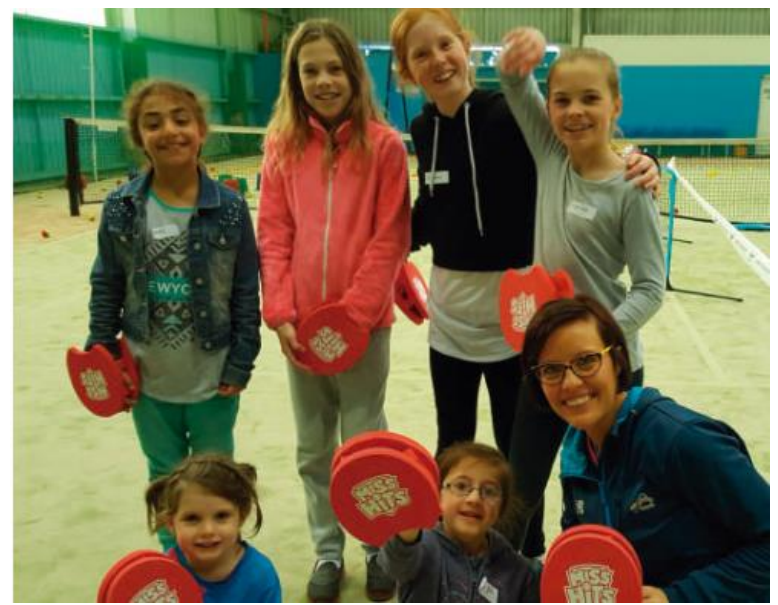

Debido a los múltiples factores que se deben tener en cuenta, se afirma que la Teoría de Liderazgo Transformacional (TLT), puede brindar un marco apropiado para evaluar las intervenciones e aumentar la participación femenina en el tenis. Partiendo de la psicología organizacional y del trabajo de Bernard Bass, se conceptualizó como una suerte de liderazgo, según el cual los seguidores son inspirados y empoderados para lograr más de lo que esperaban, debido a las conductas del líder (Bass, 1990). En la literatura de la psicología del deporte, el liderazgo transformacional demostró ser efectivo en varios deportes y contextos (Callow, Smith, Hardy, Arthur y Hardy, 2009; Price y Weiss, 2013; Smith, Arthur, Hardy, Callow, y Williams, 2013).

Además, utilizar la teoría TLT para evaluar las estrategias de participación efectiva e informar sobre las futuras, sería particularmente pertinente para las mujeres en el deporte. En un estudio reciente que explora la naturaleza de la conductas TLT exhibidas por los entrenadores que trabajan con atletas mujeres, surgieron cuatro temas asociados con las conductas TLT: afecto, motivación, enseñanza de lecciones de vida, y confianza (Newland, Newton, Podlog, Legg y Tanner, 2015). Los autores los alinearon con las conceptualizaciones originales clave de la teoría del Liderazgo Transformacional: Influencia idealizada, motivación inspiradora, estimulación intelectual, y consideración individualizada (Bass, 1985). El afecto se asoció con la consideración individualizada. Los atletas se sintieron valorizados al saber que el entrenador se preocupaba por ellos y esto se manifestó mostrando interés en ellos como persona y como jugadores. La "motivación" se relacionó con la motivación inspiradora y la estimulación intelectual, los atletas se sienten motivados por las altas expectativas físicas y mentales de su entrenador, mientras las enseñanzas de vida se vinculaban con la conceptualización TLT de la influencia idealizada. La confianza, y específicamente la confianza bidireccional, estaba también vinculada con la influencia idealizada (Newland y cols., 2015).

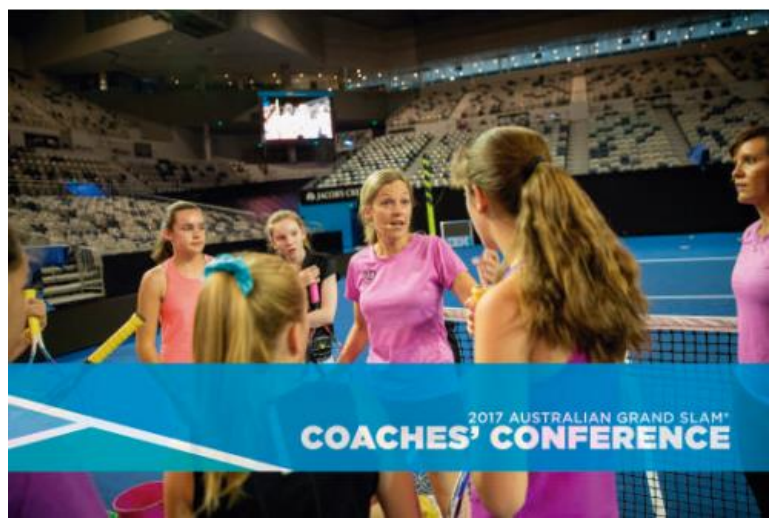

Con el fin de avanzar con la redefinición de liderazgo transformacional en el deporte, y particularmente para intentar avanzar con su pertinencia en un contexto aplicado, se desarrolló el Modelo Visión, Soporte y Desafío (Arthur y Lynn, 2016). Originalmente desarrollado en un contexto militar (Hardy, Arthur, Jones, Shariff, Munnoch, Isaacs y Allsopp, 2010) fue conceptualizado en relación con el deporte por Arthur, Hardy y Woodman (2012).

Según el modelo, los grandes entrenadores logran óptimos resultados con los atletas, proporcionando una visión inspiradora, y un equilibrio apropiado de apoyo y desafío para lograr esa visión (Arthur y cols., 2012, Arthur y Lynn, 2016). El modelo distingue entre lo que hace el entrenador y la incidencia que tienen sus conductas en los deportistas, $y$, por lo tanto, tiene el potencial de explicar el proceso de transformación que se lleva a cabo entre el entrenador y el deportista.

Se plantea la hipótesis de que el modelo VSD, sustentado como está por la teoría del liderazgo transformacional, podría proporcionar la base teórica para una herramienta de evaluación que midiera la efectividad de las intervenciones a fin de aumentar la participación deportiva.

\section{Parte Dos- Iniciativas actuales}

Judy Murray y Emma Doyle son dos entrenadoras que están liderando el camino para aumentar la participación femenina en el tenis por medio de programas transformacionales.

\section{She Rallies - Judy Murray}

She Rallies es una iniciativa de la LTA creada y encabezada por Judy Murray. La visión de She Rallies es atraer y mantener a más mujeres en el tenis, en el RU, inspirando, construyendo y empoderando a una fuerza laboral más grande y más fuerte 
con el objetivo de crear mayores oportunidades para las niñas $y$ las mujeres en el tenis.

She Rallies se inició en febrero de 2017, con una conferencia para entrenadoras de tenis solamente para mujeres y que fue la primera en su tipo. Judy y la LTA reunieron a un grupo de expertas, desde entrenadoras, y psicólogas hasta periodistas, fisiólogas y atletas paralímpicas a fin de crear una visión inspiradora del futuro del tenis femenino. Estas ponentes sirvieron un rol doble inspirando a la audiencia femenina, y siendo un modelo de rol, desafiando la idea de salir a buscar a más niñas que jueguen al tenis y a más mujeres que entreguen tenis.

En el centro del programa hay una red de Embajadoras a tiempo parcial designada para capacitar a una fuerza laboral de activistas femeninas en todo el RU. Estas embajadoras fueron capacitadas con un exhaustivo programa de entrenamiento que les permitió entregar las cuatro facetas del programa:

- Lil Miss Hits (para 5 - 8 años de edad)

- Tenis iniciante para adolescentes

- Días divertidos para niñas

- Competición recreativa para niñas

\section{Girl Power Camps - Emma Doyle}

Junto con Judy, la entrenadora de alto rendimiento australiana, Emma Doyle trabajó para lanzar el programa She Rallies. Emma y Judy se encontraron gracias a Girl Power Camps, que Emma desarrolló en Australia y se está implementando no solamente a nivel local sino también en los Estados Unidos y en varios lugares de Europa. Emma trabaja con la LTA y con Judy en la formación de entrenadoras para que puedan entrenar a las atletas de manera más efectiva, recurriendo a sus años de trabajo con jugadoras y entrenadoras y a las lecciones aprendidas trabajando en Girl Power Camps.

En el centro de Girl Power Camps se encuentran los principios de Comprometer- Empoderar- Desarrollar, que se correlacionan con las conductas TLT de afecto, motivación, enseñanza de lecciones de vida y confianza (Niewland y cols., 2015). Estos principios se utilizan para sustentar los diferentes tipos de actividades y crear una experiencia transformadora para las niñas. Los campamentos inician utilizando carteles con la visión y con actividades destinadas a aprovechar el mundo individual de las niñas y mostrarles que son atendidas. Las actividades incorporan ejercicios para motivar al grupo y construir la confianza que se desarrollará en diferentes direcciones para mostrar que el tenis, y el deporte en general, son actividades que merecen la pena, no solamente para mejorar la salud y la aptitud física, sino también para aprender valiosas lecciones para la vida. El nivel de desafío de las actividades y de los ejercicios se adapta a los niveles adecuados.

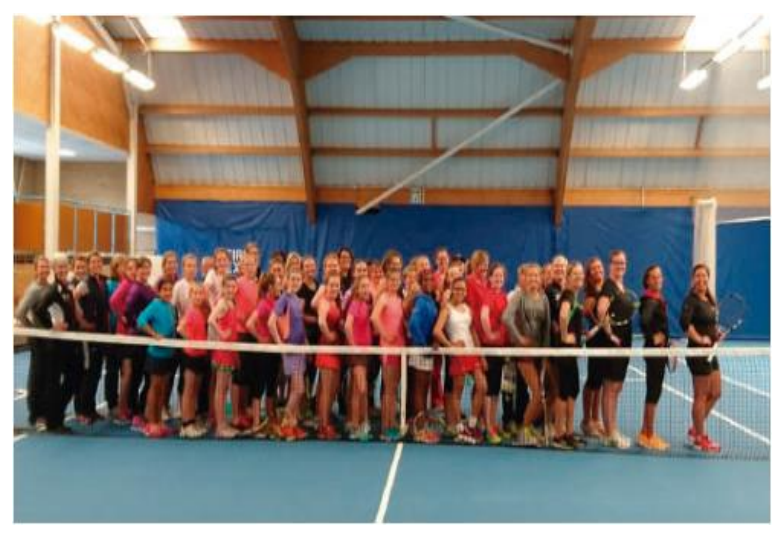

\section{CONCLUSIÓN}

Actualmente, no hay suficientes investigaciones sobre las evaluaciones de las intervenciones de entrenamiento en el deporte juvenil, que se sostienen con la teoría y se centran en lo interpersonal (Turnnidge y Côté, 2016). Además, hay escasas investigaciones sobre programas de desarrollo de entrenadoras con investigaciones informadas sobre pruebas y evaluaciones (Allan, Vierimaa, Gainforth, y Côté, 2017). Es posible que las investigaciones sobre la teoría TLT, como encuadre del programa de intervención/ desarrollo de entrenadoras para aumentar la participación de las niñas, ofrezca excelentes posibilidades, no solamente para el tenis, sino también para otros deportes

\section{REFERENCIAS}

Allan, V., Vierimaa, M., Gainforth, H. L. et Côté, J. (2017). The use of behaviour change theories and techniques in researchinformed coach development programmes: a systematic review. International Review of Sport and Exercise Psychology, 1-23. https://doi.org/10.1080/1750984X.2017.1286514

Arthur, C. A., Hardy, L. et Woodman, T. (2012). Realising the Olympic dream: Vision, support and challenge. Reflective Practice, 13(3), 399-406. https://doi.org/10.1080/14623943.2012.670112

Arthur, C. A. et Lynn, A. (2016). Transformational leadership and the role of the coach. The Psychology of Sports Coaching: Research and Practice, 187. https://doi.org/10.4324/9781315689210-14

Bass, B. M. (1985). Leadership and performance beyond expectations. Collier Macmillan.

Bass, B. (1990). Handbook of leadership: Theory, research, and managerial applications (3e éd.). New York, NY: Free Press. https://doi.org/10.1016/1048-9843(90)90016-B

Biddle, S. J., Braithwaite, R. et Pearson, N. (2014). The effectiveness of interventions to increase physical activity among young girls: $A$ meta-analysis. Preventive Medicine, 62, 119-131. https://doi.org/10.1016/j.ypmed.2014.02.009

Davies, S., Burns, H., Jewell, T. et McBride, M. (2011). Start active, stay active: a report on physical activity from the four home countries. Chief Medical Officers, 16306, 1-62. 
Hardy, L., Arthur, C. A., Jones, G., Shariff, A., Munnoch, K., Isaacs, I. et Allsopp, A. J. (2010). The relationship between transformational leadership behaviors, psychological, and training outcomes in elite military recruits. The Leadership Quarterly, 21(1), 20-32. https://doi.org/10.1016/j.leaqua.2009.10.002

Inchley, J., Mitchell, F. et Currie, C. (2012). Fit for Girls Evaluation: Final Evaluation Report.

Nader, P. R., Bradley, R. H., Houts, R. M., McRitchie, S. L. et O'Brien, M. (2008). Moderate-to-vigorous physical activity from ages 9 to 15 years. Jama, 300(3), 295-305. https://doi.org/10.1001/jama.300.3.295

Newland, A., Newton, M., Podlog, L., Legg, W. E. et Tanner, P. (2015). Exploring the nature of transformational leadership in sports: a phenomenological examination with female athletes. Qualitative Research in Sport, Exercise and Health, 7(5), 663- 687. https://doi.org/10.1080/2159676X.2015.1007889

Price, M. S. et Weiss, M. R. (2013). Relationships among coach leadership, peer leadership, and adolescent athletes' psychosocial and team outcomes: A test of transformational leadership theory. Journal of applied sport psychology, 25(2), 265-279. https://doi.org/10.1080/10413200.2012.725703

Priest, N., Armstrong, R., Doyle, J. et Waters, E. (2008). Interventions implemented through sporting organisations for increasing participation in sport. The Cochrane Library. https://doi.org/10.1002/14651858.CD004812.pub3

Reading, A. V. et Hansson. (2014). This Girl Can.

Smith, M. J., Arthur, C. A., Hardy, J., Callow, N. et Williams, D. (2013). Transformational leadership and task cohesion in sport: The mediating role of intrateam communication. Psychology of Sport and Exercise, 14(2), 249-257. https://doi.org/10.1016/j.psychsport.2012.10.002

Sport England (2015). Go where women are: Insight on engaging women and girls in sport and exercise. London: Sport England.
Turnnidge, J. et Côté, J. (2016). Applying transformational leadership theory to coaching research in youth sport: A systematic literature review. International Journal of Sport and Exercise Psychology,

$1-16$. https://doi.org/10.1080/1612197X.2016.1189948

CONTENIDO ITF ACADEMY RECOMENDADO (HAZ CLICK ABAJO)

\section{ITF Academy}

Derechos de Autor (c) 2017 Julie Gordon, Judy Murray y Emma Doyle

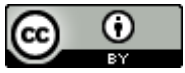

Este texto está protegido por una licencia CreativeCommons 4.0 .

Usted es libre para Compartir -copiar y redistribuir el material en cualquier medio o formato- y Adaptar el documento -remezclar, transformar y crear a

partir del material- para cualquier propósito,, incluso para fines comerciales, siempre que cumpla la condición de:

Atribución: Usted debe dar crédito a la obra original de manera adecuada, proporcionar un enlace a la licencia, e indicar si se han realizado cambios. Puede hacerlo en cualquier forma razonable, pero no de forma tal que sugiera que tiene el apoyo del licenciante o lo recibe por el uso que hace de la obra.

$\underline{\text { Resumendelicencia - Textocompletodelalicencia }}$ 